\title{
Lack of Increase in Intracranial Pressure After Epidural Blood Patch in Spinal Cerebrospinal Fluid Leak
}

\author{
Jens Fichtner • Christian Fung • Werner Z'Graggen • \\ Andreas Raabe $\cdot$ Jürgen Beck
}

Published online: 17 April 2012

(C) Springer Science+Business Media, LLC 2012

\begin{abstract}
Background and Importance Epidural blood patch (EBP) is one therapeutic measure for patients suffering from spontaneous intracranial hypotension (SIH) or post-lumbar puncture headaches. It has been proposed that an EBP may directly seal a spinal cerebrospinal fluid (CSF) fistula or result in an increase in intracranial pressure (ICP) by a shift of CSF from the spinal to the intracranial compartment. To the best of our knowledge this is the first case of a patient with SIH and neurological deterioration in whom ICP was measured before, during, and after spinal EBP.

Clinical Presentation This 52-year old previously healthy man presented with holocephal headaches. MRI showed a left hemispheric subdural fluid collection causing a significant mass effect. Myelography revealed a CSF leak with epidural contrast at the left side of the L-2 level. To seal the CSF leak, we performed an EBP procedure targeted at left L-2 level and recorded ICP. After applying the epidural blood patch $(15 \mathrm{cc})$ the patient improved rapidly, ICP however remained unchanged before, during, and after the procedure. One day post-treatment, he had a GCS score increase from 12 to 15 and no headache or neurological deficits.

Conclusion A shift of CSF from the spinal to the cranial compartment with a subsequent rise in ICP might not be a
\end{abstract}

J. Fichtner · C. Fung · W. Z'Graggen · A. Raabe · J. Beck ( $₫)$

Department of Neurosurgery, Bern University Hospital,

Publications Office, 3010 Bern, Switzerland

e-mail: juergen.beck@insel.ch beneficial therapeutic mechanism of spinal epidural blood patching.

Keywords Spontaneous intracranial hypotension . Cerebrospinal fluid leakage - Epidural blood patch . Intracranial pressure

\section{Introduction}

Spontaneous intracranial hypotension (SIH) is an increasingly recognized syndrome with the hallmark symptom of orthostatic headache. This syndrome is caused by spontaneous spinal dural tears and loss of cerebrospinal fluid (CSF) [1]. After lumbar puncture or spinal surgery, a clinically similar syndrome results from continued loss of CSF: post-lumbar puncture headaches. If resolution of symptoms after a short period of bed rest fails, another therapeutic option is to apply a spinal epidural blood patch (EBP). In the case that the spinal dural tear or iatrogenic bore is at the level of the blood patch, the instilled blood may directly seal the hole, stop CSF loss and improve the symptoms [2-4]. Even when the exact site of the spinal dural tear is unknown or distant to the site of blood patch application (usually the lumbar level or thoraco-lumbar junction), the procedure may immediately alleviate patient symptoms [5-7]. The most likely explanation for this mechanism is that the intraspinal CSF compartment becomes compressed and CSF is shifted from the spinal to the intracranial compartment. If there is indeed a shift of CSF and this would be the therapeutic mechanism, we hypothesize that one should be able to measure changes, i.e., an increase of intracranial pressure (ICP) during or shortly after epidural blood patching. Here, we present our findings of a patient in whom we measured ICP before, during, and after epidural blood patching. 


\section{Case Report}

Presentation and Examination

This 52-year old previously healthy man presented to the emergency department with holocephal headaches (for approximately 2 months). There was no orthostatic component of the headache described by the patient although his wife described a behavior change and memory decline. Magnetic resonance imaging (MRI) showed a left hemispheric subdural fluid collection causing a significant mass effect (Fig. 1). Further investigation of his medical history revealed no trauma, head injury or oral anticoagulants. The initial examination showed a Glasgow Coma Scale (GCS) score of 15 with some short-term memory deficits and a perseveration tendency.

\section{Initial Course}

The diagnosis was a left-sided chronic subdural hematoma. In the initial surgery, we identified a hematoma with a typical "motor oil" appearance. After treatment by two burr hole trephination, the patient developed multiple recurrences, declined neurologically, and had to be intubated due to decreased consciousness. We ruled out metabolic causes and started an early seizure prophylaxis with oxcarbazepine to treat complex partial seizures or a possible status epilepticus. Serial brain scans showed no

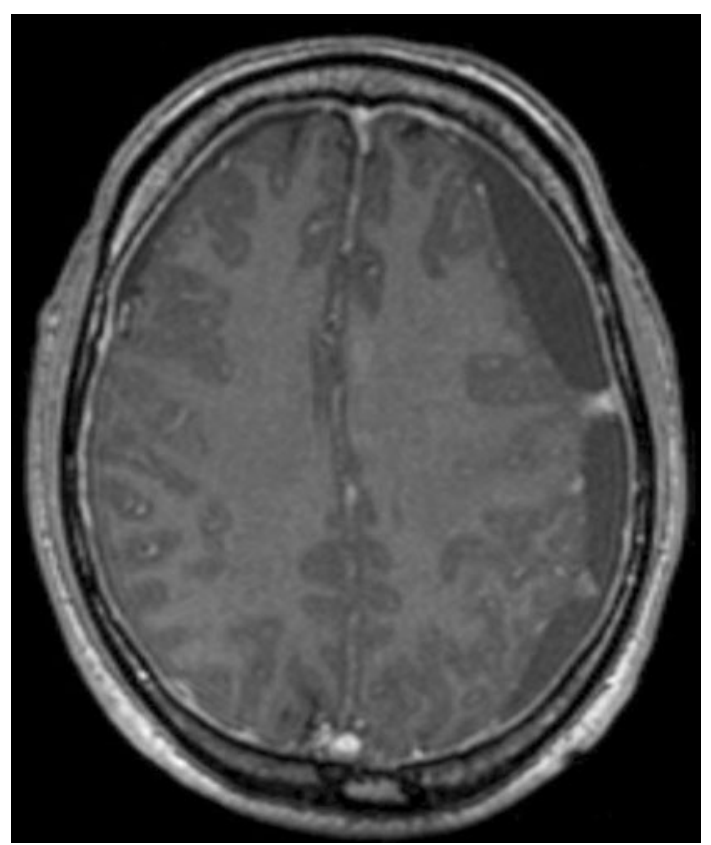

Fig. 1 Initial T1-MRI scan on admission of the patient. Left-sided subdural hematoma can be observed

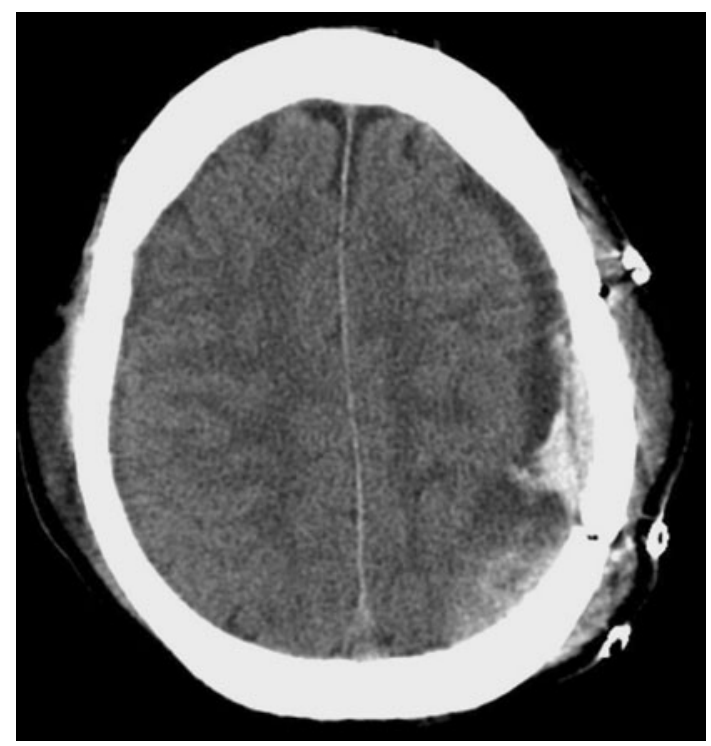

Fig. 2 CT scan showing recurrent subdural hematoma before third surgery

other pathology than recurrent subdural hematoma (Fig. 2). The patient underwent three operations for the subdural hematoma, including osteoplastic trepanation. In the third surgery, we inserted a subdural pressure probe (Spiegelberg, Hamburg, Germany) for ICP measurement. Myelography revealed a CSF leak with epidural contrast at the left side of the $\mathrm{L}-2$ level. The patient was then transferred to the intensive care unit and ICP was recorded. Although the patient was not under sedation, his GCS decreased 3 points to 12 (Eyes 3, Verbal 3, Motor 6) and his level of consciousness decreased. There were no motor or pupillary changes. To seal the CSF leak we performed an EBP procedure targeted at left L-2 level and recorded ICP before, during, and after the procedure.

\section{Results of ICP Recording}

We recorded events at 2-min intervals before and after epidural blood patching using a continuous ICP data recording system (Patient Data Management System, GE Healthcare, Zurich, Switzerland). Two hours before applying the EBP, the patient was lying flat in a supine position and showed a stable ICP of $5.6 \pm 2.3 \mathrm{mmHg}$ (mean \pm standard deviation). Sedation was not necessary during the procedure. When the patient was positioned from a supine to a left lateral position, the ICP rose to $8.4 \pm 0.9 \mathrm{mmHg}$. Ten minutes after local anesthesia was administered, the ICP was exactly $10.0 \mathrm{mmHg}$. From this point we could establish a stable ICP recording (starting point for time scale in Fig. 3a). For the next 7 min, the ICP was $10.5 \pm 0.6 \mathrm{mmHg}$ (Table 1; Fig. 3a). During this time $15 \mathrm{ml}$ autologous blood was taken. Two minutes after the blood was taken, an EBP procedure was performed with 
a Intracranial pressure by subdural pressure probe in high resolution

\section{ICP in}

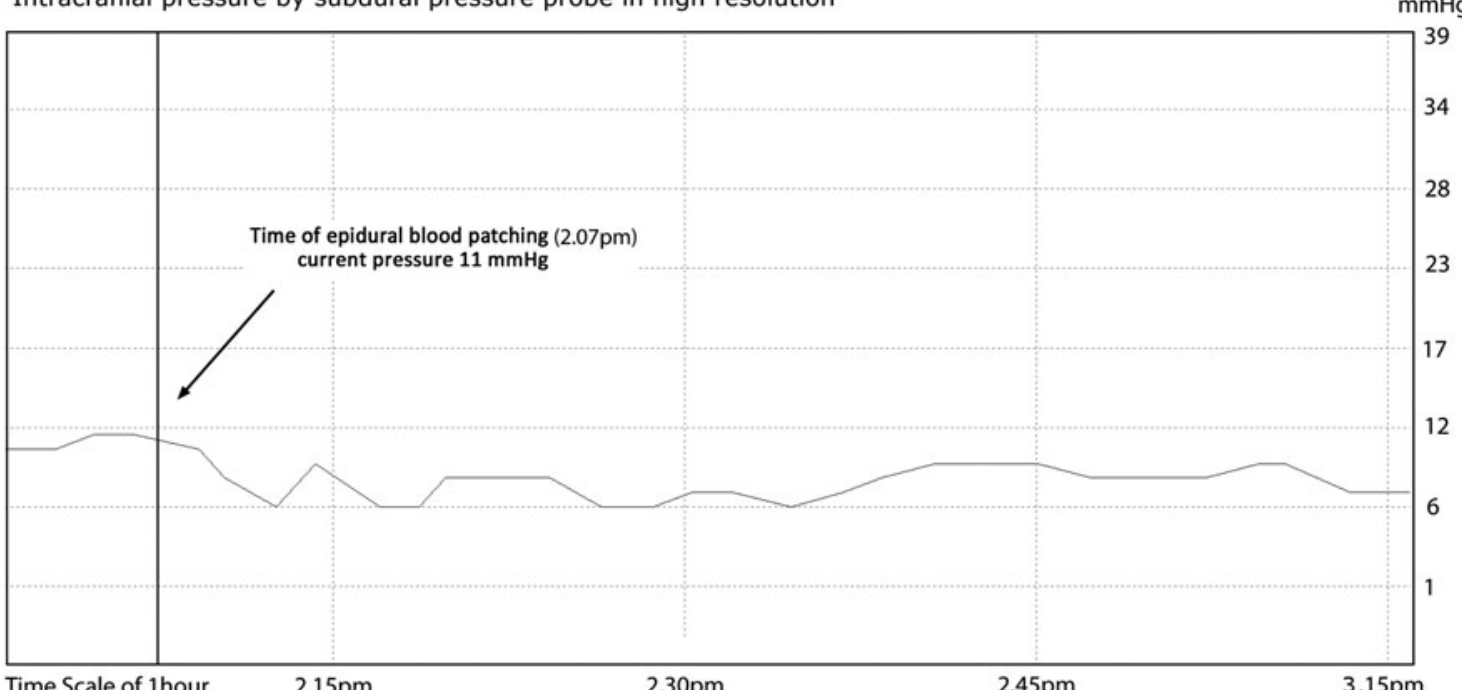

Time Scale of 1hour

$2.15 \mathrm{pm}$

$2.30 \mathrm{pm}$

$2.45 \mathrm{pm}$

$3.15 \mathrm{pm}$

\section{b}

Intracranial Pressure in $\mathrm{mmHg}$ measured by a subdural pressure probe in a $5 \mathrm{~min}$. resolution

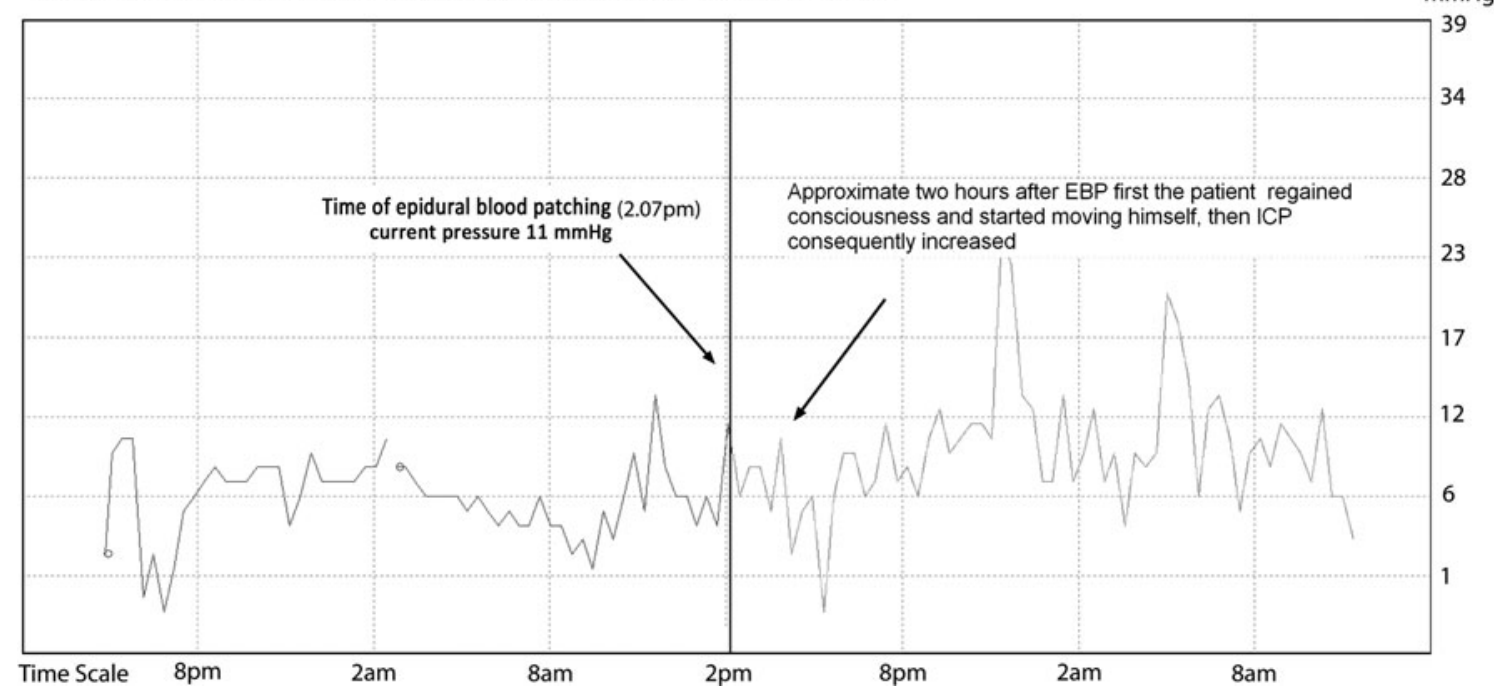

Fig. 3 a ICP measurement immediately before, during and after EBP procedure plus a $1 \mathrm{~h}$ period after EBP in 2-min intervals (the black solid line is representing the epidural blood patch procedure). $\mathbf{b}$ ICP

measurement $21 \mathrm{~h}$ before and after EBP in a 5-min interval (the black solid line is representing the epidural blood patch procedure)

injection of $15 \mathrm{ml}$ autologous blood in the L1-L2 level for about $1 \mathrm{~min}$. At the start of the EBP, the ICP was $11 \mathrm{mmHg}$. During the procedure, the mean ICP was $8.8 \pm 1.9 \mathrm{mmHg}$. Seven minutes later, the patient was positioned back to supine position and ICP was $9 \mathrm{mmHg}$. During the initial $69 \mathrm{~min}$ after EBP procedure, ICP decreased significantly to $7.8 \pm 1.2 \mathrm{mmHg}$, compared with the stable readings after patient positioning and before EBP $(p<0.0001$ unpaired $t$-test; Fig. 3a; Table 1). After 69 min the patient started moving himself and regained consciousness. At this point, the ICP values were no longer stable as previously observed when the patient was lying in supine position. With continuous ICP measurement, no significant increase of ICP was observed within the first and second hour after EBP even when the patient regained conscious after $69 \min (p=0.19$; unpaired $t$-test $)$. The mean ICP was $7.6 \pm 1.1 \mathrm{mmHg}$ in the first hour and $7.1 \pm 2.2 \mathrm{mmHg}$ in the second hour after EBP, compared with $10.5 \pm 0.6 \mathrm{mmHg}$ before EBP (Table 1). Over the following $2 \mathrm{~h}$, the patient's condition improved rapidly. The patient regained consciousness and started moving himself to the prone position, his GCS score increased to 15 and consequently, his ICP also increased (Fig. 3b; Table 1). 
Table 1 Time periods and mean values of ICP measured with the intracranial pressure probe before and after EBP on Fig. $3 \mathrm{a}$, b as well as a comparison of ICP values in the first and second hour after EBP

\begin{tabular}{lll}
\hline Comparison of ICP values before and after EBP & Time & ICP in mmHg (mean \pm standard deviation) \\
\hline Time before EBP on Fig. 3a & 7 min before EBP & $10.5 \pm 0.6$ \\
Time after EBP on Fig. 3a & 69 min after EBP & $7.8 \pm 1.2$ \\
The two-tailed $P$ value $<0.0001^{*}$ & $21 \mathrm{~h} 15 \mathrm{~m}$ before EBP & $6.0 \pm 2.4$ \\
Time before EBP in Fig. 3b & $21 \mathrm{~h} 15 \mathrm{~m}$ after EBP & $9.1 \pm 4.2$ \\
Time after EBP on Fig. 3b & & $7.6 \pm 1.1$ \\
The two-tailed $P$ value $<0.0001^{*}$ & First hour after EBP & $7.1 \pm 2.2$ \\
ICP first hour after EBP & Second hour after EBP & \\
ICP second hour after EBP & & \\
The two-tailed $P$ value $=0.19^{*}$ & &
\end{tabular}

ICP Intracranial pressure, ICP was measured with an intracranial subdural probe

$* P$-value was considered statistically significant when $P<0.05$ using an unpaired $t$-test

\section{Recovery, Outcome}

One day post-treatment, the GCS score remained at 15 with no headache or neurological deficits. The ICP probe was removed $21 \mathrm{~h}$ after the EBP procedure. A MRI scan 5 days after EBP showed a regressive hematoma with only a small residual subdural fluid collection (Fig. 4). Two weeks later at the follow-up visit, there were no clinical signs of intracranial hypotension. The patient had returned to work and socially reintegrated. A clinical follow-up with an MRI scan 6 months after EBP showed no signs of recurrent hematoma (Fig. 5).

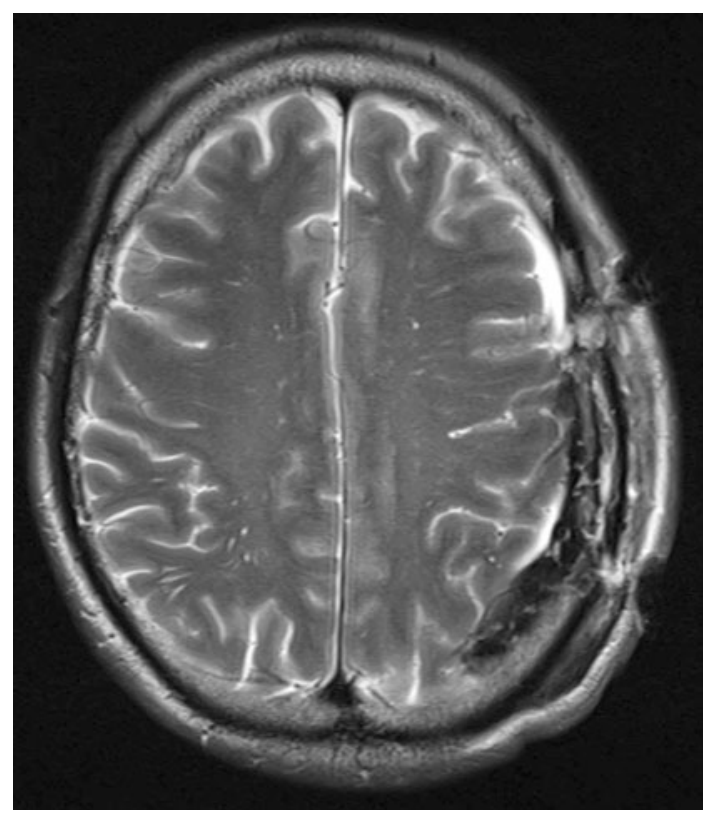

Fig. 4 MRI T2 scan showing the subsequent result 5 days after EBP

\section{Discussion}

Epidural blood patch for the treatment of CSF leaks in SIH seems to be a safe and effective treatment [3, 4, 8-11]. Some authors estimate an ICP normalization after epidural blood patch [11], however, to the best of our knowledge, continuous ICP monitoring has not been described yet. In our patient, the ICP was measured with a subdural pressure probe that was implanted during the third hematoma evacuation, 1 day before the EBP procedure. The ICP measurements before, during, and after EBP did not show an increase of ICP.

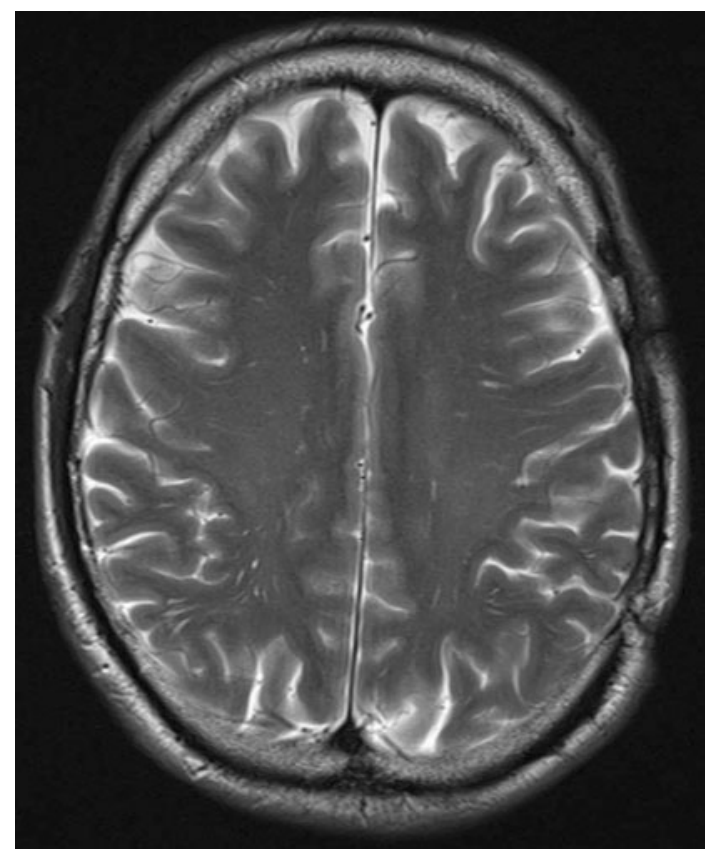

Fig. 5 MRI T2 scan showing the results of EBP 6 months after surgery 
One major argument used to explain a potential mechanism of epidural fluid injection is the shift of CSF and increase of ICP, however, this hypothesis is made without collection of ICP measurements. In 1967, Usubiaga et al. [7] examined the effect of epidural saline injections for the treatment of postspinal anesthesia headaches. In this study, they measured the spinal subarachnoid and spinal epidural pressure after injecting 10 or $20 \mathrm{ml}$ of saline solution into the epidural space at the lumbar level. Pressure was recorded for $10 \mathrm{~min}$. A peak was reached immediately after the injection with an increase of $65 \mathrm{~cm} \mathrm{H}_{2} \mathrm{O}$ epidurally and up to $85 \mathrm{~cm} \mathrm{H}_{2} \mathrm{O}$ subarachnoidally. A return to previous values was observed within 3-10 min. There was no ICP measurement. The authors did agree that compression of the spinal dura by saline solution can account for an immediate relief but the effect was too transient to explain the prolonged headache alleviation. This is concordant to our observations that EBP does not lead to increased pressure intracranially. Furthermore, the authors also discussed another more permanent sealing of the dural opening, such as the formation of a fibrin clot or a prolapse of the arachnoid. This can explain why instilled blood travels along the epidural space and seals the bore.

Sencakova et al. [6] assumed an early and a long-lasting effect for the efficacy of EBP in spontaneous CSF leaks. The early effect, within minutes, may consist of a displacement of the dura toward the spinal cord and cauda equine, resulting in a decrease of the CSF volume thereby compensating for low ICP. The long-lasting effect is due to a sealing of the dural leak. The "Plug" theory (1999) described by Duffy et al. [2], assumed that injected blood forms a gelatinous plug that seals the hole and prevents further CSF leakage into the epidural space. The regeneration of the CSF restores the ICP and alleviates the headache, then normal reparative responses seal the dural hole. This was based on the laboratory investigations of DiGiovanni et al. [12] who described reparative processes of the dura with engulfment of immature fibroblasts as a long lasting effect. But this theory cannot account for patients who obtain immediate relief after blood patching. Due to an increase of the epidural pressure, the spinal CSF is displaced to the cranium immediately, restoring intracranial volume and pressure. As with the Usubiaga et al. study, Duffy et al. did not collect any ICP measurements. The authors describe the influence of "multiple mechanisms" as providing headache relief in EBP [2].

Recently Franzini et al. [5] assumed that a dural leak is not the necessary cause of the disease. The theory is based on anatomical considerations about the spinal venous drainage system. SIH syndrome is the effect of epidural hypotension maintained by the inferior cava vein outflow to the heart. The goal of the blood patch procedure was not to seal CSF leaks, but instead to help in reversing a pathological CSF blood gradient within the epidural space along the entire cord [5]. However, no pressure (neither ICP nor spinal lumbar, thoracic or cervical, nor in the vena cava) was measured in their work.

One limitation to our work was that the subdural ICP measurement may not have been sensitive enough to measure ICP changes triggered by EBP, although we could establish reliable measurements and the ICP probe was reactive to jugular compression testing. It is unlikely that very subtle changes in ICP would result in such a profound improvement of patient's symptoms as reported here or in many other cases.

\section{Conclusion}

Despite the usefulness of EBP, the mechanisms are still not clearly identified. Although several explanations have been made, a combination of pathophysiological events is probably responsible for the effect of EBP. An elevation of ICP is not essential to explain the efficacy of EBP. We could not measure any increase of ICP directly or in the hours after EBP. In all these possible explanations, an acute (seconds to several minutes) or subacute (hours) increase of ICP may not play a key role in the treatment of SIH.

Acknowledgments The final draft of the manuscript was proofread by Susan Wieting, Bern University Hospital, Publications Office, Bern Switzerland.

\section{References}

1. Schievink WI, Meyer FB, Atkinson JL, et al. Spontaneous spinal cerebrospinal fluid leaks and intracranial hypotension. J Neurosurg. 1996;84:598-605.

2. Duffy PJ, Crosby ET. The epidural blood patch. Resolving the controversies. Can J Anaesth. 1999;46:878-86.

3. Rai A, Rosen C, Carpenter J, et al. Epidural blood patch at C2: diagnosis and treatment of spontaneous intracranial hypotension. AJNR Am J Neuroradiol. 2005;26:2663-6.

4. Wang J, Wang L. Targeted epidural blood patch: an effective treatment for spontaneous intracranial hypotension. Zhonghua Yi Xue Za Zhi. 2009;89:1477-9.

5. Franzini A, Messina G, Nazzi V, et al. Spontaneous intracranial hypotension syndrome: a novel speculative physiopathological hypothesis and a novel patch method in a series of 28 consecutive patients. J Neurosurg. 2010;112:300-6.

6. Sencakova D, Mokri B, McClelland RL. The efficacy of epidural blood patch in spontaneous CSF leaks. Neurology. 2001; 57:1921-3.

7. Usubiaga JE, Usubiaga LE, Brea LM, et al. Effect of saline injections on epidural and subarachnoid space pressures and relation to postspinal anesthesia headache. Anesth Analg. 1967; 46:293-6.

8. Su CS, Lan MY, Chang YY, et al. Clinical features, neuroimaging and treatment of spontaneous intracranial hypotension and magnetic resonance imaging evidence of blind epidural blood patch. Eur Neurol. 2009;61:301-7. 
9. Taillia H, Meyer X. Indications for the use of an epidural blood patch in the treatment of spontaneous intracranial hypotension. Rev Neurol (Paris). 2003;159:1181-5.

10. Waguri N, Tomita M, Hayatsu K, et al. Epidural blood patch for treatment of spontaneous intracranial hypotension. Acta Anaesthesiol Scand. 2002;46:747-50.

11. Zada G, Pezeshkian P, Giannotta S. Spontaneous intracranial hypotension and immediate improvement following epidural blood patch placement demonstrated by intracranial pressure monitoring. Case report. J Neurosurg. 2007;106:1089-90.

12. DiGiovanni AJ, Galbert MW, Wahle WM. Epidural injection of autologous blood for postlumbar-puncture headache. II. Additional clinical experiences and laboratory investigation. Anesth Analg. 1972;51:226-32. 